\title{
Sérotypes de Salmonella isolés chez les petits ruminants abattus à Dakar
}

\author{
par M. P. DOUTRE $(*)$ et R. BOCHE $(* *)$ \\ avec la collaboration technique de B. TOURE $(* * *)$ et A. TALL $(* * *))$
}

\begin{abstract}
RÉSUMÉ
Une enquête sur le portage chronique de Salmonella chez les petits ruminants sacrifiés à l'abattoir de Dakar est effectuée.

Les ganglions mésentériques de 1108 ovins et de 1018 caprins sont recueillis. Après enrichissement en milieu sélénite, 90 souches sont isolées appartenant à 52 sérotypes. 3 sérotypes nouveaux sont découverts $: S$. derklé, $S$. lodé et $S$. bignona et 3 sérotypes rapportés pour la première fois au Sénégal : $S$. lawra, $S$. ruiru et $S$. tornow. Le taux d'infection chez le mouton s'établit à 4,7 p. 100 et chez la chèvre à 3,6 p. 100 . Ces résultats sont voisins de ceux obtenus dans d'autres pays africains.

L'incidence sur l'hygiène publique du portage chronique de Salmonella chez les petits ruminants est discutée.
\end{abstract}

Le portage chronique de Salmonella chez les petits ruminants a déjà fait l'objet d'enquêtes épidémiologiques dans différents pays. En Afrique, les conclusions des auteurs s'opposent bien souvent : au Nigeria, JOHNSON, en 1958 (9), ne rencontre aucun porteur après examen de 40 moutons et 90 chèvres; à l'abattoir de Kano, OLA OJO, en 1974 (15), n'isole aucune Salmonella à partir de 375 prélèvements caprıns ; par contre, au Ghana, ZWART, en 1962 (22), met en évidence un taux d'infection de 3,7 p. 100 chez le mouton et de $5,6 \mathrm{p}$. $100 \mathrm{chez}$ la chèvre après étude de 80 prélèvements provenant de chacune de ces espèces ; ceci rejoint les anciens résultats de VAN OYE, DEOM, VERCRUYSSE et FASSEUX obtenus à Elisabethville en 1957 ; là, sur 2137 animaux soumis à l'analyse bactériologique, 5,5 p. 100 des moutons et 2,4 des

(*) Chef du Service de Bactérıloge du Laboratoire de l'Elevage et de Recherches vétérinaires (I. S. R. A.), B. P. 2057, Dakar, Sénégal.

(**) Chef du Service de Bactériologie de l'Instıtut Pasteur de Dakar.

(***) Technicien supérieur et ande biologiste au Laboratoire national de l'Elevage et de Recherches vétérinaires (I. S. R. A.). chèvres se révélèrent porteurs de Salmonella. Au Soudan, en 1970, KHAN (10), après étude de 1750 prélèvements ovins et de 500 caprins, trouve des taux d'infection de 3,77 et 1 p. 100 selon les hôtes. L'étude du portage chronique de Salmonella aboutit parfois à l'isolement de sérotypes nouveaux; ainsi au Tchad, en 1963 (12), Salmonella mara (39; e, h ; - ) et S. meskin $(51: \mathrm{e}, \mathrm{h}: 1,2)$ sont décrites pour la première fois. En Asie, des travaux analogues ont été poursuivis : KUMAR, SAXENA et GUPTA, aux Indes, en 1973 (11), examinent 812 moutons et 683 chèvres et isolent 72 souches appartenant à 22 sérotypes : à la même époque et toujours aux Indes, JANAKIRAMAN et RAJENDRA (8) étudient le portage des chèvres utilisées pour la production du vaccin lyophilisé contre la peste bovıne et découvrent après examen de 1931 prélèvements que 14 p. 100 des animaux sont porteurs de germes. En Iran, TADJEBAKHCHE et NAMIN, en 1974 (14, 17), trouvent chez la chèvre un taux d'infection moindre mais signalent la présence de $S$. anatum et $S$. derby.

Au Sénégal, le portage de Salmonella par 
différentes espèces animales, domestiques ou non, a donné lieu à plusieurs travaux au cours des dix dernières années. Le Laboratorre national de l'Élevage et de Recherches vétérinaires de Dakar et l'Institut Pasteur se sont ainsi attachés à déceler, grâce à des enquêtes systématiques, l'existence du germe chez le porc (2), les rapaces anthropophiles (1) et les chiroptères (4), accidentellement des souches ont été obtenues d'autres oiseaux, de reptiles, etc...
Dans un avenir proche, une mise à jour de l'ensemble des sérotypes rencontrés dans le pays, chez l'animal, sera effectuée (environ 150 sérotypes à ce jour).

C'est dans ce cadre général qu'une étude a été entreprise, en 1974 et 1975, sur les petits ruminants sacrifiés à l'abattoir de Dakar. La proximité de cet établissement a permis des récoltes en général bi-hebdomadaires d'un matériel abondant.

TABLEAU $N^{\circ}$ I - Sërotypes de SalmonęZa isolës chez le mouton (ganglion mésentërique)

\begin{tabular}{|c|c|c|c|c|}
\hline $\begin{array}{c}\text { Groupe } \\
\text { sérologique }\end{array}$ & Sérotype & Fornule antigënique & $\begin{array}{l}\text { Nombre de } \\
\text { souches }\end{array}$ & $\begin{array}{c}\text { Isolement chez } \\
1^{\prime} \text { homme }\end{array}$ \\
\hline \multirow[t]{5}{*}{ B } & S. brandenburg & $1,4,12 ; 1, v ; e, 0,215$ & 2 & Tomme \\
\hline & s. bredeney & $\underline{1}, 4,12, \underline{27} ; 1, v ; 1,7$ & 4 & $"$ \\
\hline & S. twohimurium & $\underline{1}, 4,(5), 12 ; i ; I, 2$ & 2 & $"$ \\
\hline & S. reading & $1,4,(5), 12 ; e, h ; I, 5$ & I & $"$ \\
\hline & S. chester & $\underline{l}, 5,(5), 12 ; e, h ; e, n, x$ & 2 & $"$ \\
\hline \multirow[t]{5}{*}{$\mathrm{C} 1$} & S. Virchow & 6,$7 ; r ; I, 2$ & 3 & $"$ \\
\hline & 5. tenreasee & 6,$7 ; z 29 ;-$ & 1 & $"$ \\
\hline & S. isangi & 6,$7 ; d ; I, 5$ & 1 & $"$ \\
\hline & S. denver & 6,$7 ; a ; e, n, z 15$ & I & \\
\hline & S. somone & 6,$7 ; z 4, z 24 ;-$ & 1 & $"$ \\
\hline C3 & S. kentucky & (8). $20 ; i ; z 6$ & 2 & " \\
\hline \multirow[t]{3}{*}{ DT } & s. durban & 9,$12 ; a ; e, n, 215$ & 3 & Ir \\
\hline & S. enteritidis & $1,9,12 ; \mathrm{g}, \mathrm{m} ;(1,7)$ & 1 & $"$ \\
\hline & S. eastboume & $\underline{1}, 9,12 ; \mathrm{e}, \mathrm{h} ; \mathrm{I}, 5$ & 1 & $" 1$ \\
\hline \multirow[t]{3}{*}{ EI } & S. give & 3,$10 ; 1, v ; I, 7$ & 1 & $"$ \\
\hline & S. oxford & 3,$10 ; a, 1,7$ & 2 & $" 1$ \\
\hline & S. menster & 3,$10 ; e, h ; I, 5$ & 1 & 11 \\
\hline E2 & S. new-bmonswiak & 3,$15 ; 1, v ; I, 7$ & 1 & $1 "$ \\
\hline E4 & S. rigor & $1,3,19 ; 1, v ; I, 5$ & 1 & $1 "$ \\
\hline F & S. mibistaw & $11 ; r ; e, n, x$ & 2 & $"$ \\
\hline G1 & S. friedenau & 13,$22 ; d ; I, 6$ & 1 & 11 \\
\hline \multirow[t]{3}{*}{ G2 } & S. havana & $\underline{1}, 13,23 ; f, g,(s) ;-$ & 1 & $"$ \\
\hline & S. grumpensis & 13,$23 ; d ; I, 7$ & 1 & $" 1$ \\
\hline & S. vel el kebir & 13,$23 ; d ; e, n, z 15$ & 1 & $"$ \\
\hline $\mathrm{H}$ & S. caracas & $1,6,14,25 ; 8$, m, s;- & 1 & $"$ \\
\hline$I$ & S. welikade & $16 ; 1, v ; I, 7$ & 1 & $n$ \\
\hline $\mathrm{J}$ & S. carmez & $17 ; 1, v ; e, n, x$ & 1 & \\
\hline \multirow[t]{2}{*}{$\mathcal{L}$} & S. ruiru & $21 ; y ; \epsilon, \mathrm{n}, \mathrm{x}$ & 1 & \\
\hline & S. minnesota & $21 ; b ; e, n, x$ & 1 & " \\
\hline \multirow[t]{2}{*}{$\mathrm{N}$} & S. urbana & $30 ; b ; e, n, x$ & 1 & $"$ \\
\hline & S. bietri & $30 ; y ; I, 5$ & 1 & $"$ \\
\hline $\mathbf{P}$ & S. thiaroye & $38 ; e, h ; I, 2$ & 1 & 11 \\
\hline \multirow[t]{2}{*}{$\mathbf{R}$} & S. tilene & I, $40 ; \mathrm{e}, \mathrm{h} ; \mathrm{I}, \mathrm{2}$ & 2 & IT \\
\hline & S. johannesburg & 1,$40 ; b ; e, n, x$ & 1 & $"$ \\
\hline $\mathrm{S}$ & S. wayoross & $41 ; z 4, z 23 ;-$ & 4 & " \\
\hline $\mathrm{W}$ & S. apcea & $45 ; \pi, t ;-$ & 1 & \\
\hline 52 & S. derikle & $52 ; \mathrm{e}, \mathrm{h} ; \mathrm{I}, 7$ & 1 & \\
\hline
\end{tabular}




\section{MATÉRIEL ET MÉTHODE}

\section{A. Matériel d'étude}

KHAN a montré que chez les petits ruminants, la mise en culture des ganglions mésentériques permettait l'isolement d'une quantité maximale de souches, l'ensemencement de la bile ou du jus de rumen donnant des résultats nettement inférieurs (10). La présente étude repose donc uniquement sur l'utilisation des ganglions mésentériques. Ceux-ci sont recueillis stérilement après ouverture de la cavité abdominale au moment de l'éviscération. Les risques de contamination par le couteau du boucher, quoique très faibles, demeurent toujours possibles. Ce fait peut éventuellement fausser les résultats par excès. Les prélèvements sont rapportés au Laboratoire où ils sont immédiatement traités. Dans ces conditions, 1108 ganglions mésentériques de mouton et 1018 gan- glions mésentériques de chèvres sont soumis à la technique d'isolement.

\section{B. Méthodes}

Les opérations classiques suivantes sont effectuées :

- broyage du prélèvement ;

- enrichissement pendant 48 heures en bouillon sélénite ;

— isolement sur milieu sélectif (désoxycholate-citrate-lactose);

- étude des caractères des colonies suspectes (milieu urée-indole, milieu de Kliger, mannitol mobilité, recherche de la beta galactosidase) ;

- sérologie de groupe.

Certaines agglutinations flagellaires délicates ne pouvant être réalisées à Dakar, les souches, centralisées au Centre national sénégalais des

TABLEAU $N^{\circ} I I$ - Sérotypes de Salmonella isolés chez la chèvre (ganglion mésentếrique)

\begin{tabular}{|c|c|c|c|c|}
\hline $\begin{array}{c}\text { Groupe } \\
\text { sêrologique }\end{array}$ & Sêrotype & Formule antigénique & $\begin{array}{l}\text { Nombre de } \\
\text { soucbes }\end{array}$ & $\begin{array}{c}\text { Isolement chez } \\
\text { I'homme }\end{array}$ \\
\hline \multirow[t]{3}{*}{ B } & S. bredeney & $\underline{1}, 4,12, \underline{27} ; 1, v ; 1,7$ & 1 & Honme \\
\hline & S. chester & $\underline{1}, 5,(5), 12 ; e, h ; e, n, x$ & 2 & $"$ \\
\hline & S. & $1,4,12,27 ; 1, v ;-$ & 2 & \\
\hline \multirow[t]{5}{*}{ CI } & S. virenow & 6,$7 ; r ; I, 2$ & 1 & $"$ \\
\hline & S. montevideo & 6,$7 ; g, m,(p), 5 ;-$ & 2 & $"$ \\
\hline & S. redba & 6,$7 ; z 10 ; z 6$ & 1 & \\
\hline & S. orconienburg & 6,$7 ; m, t ;-$ & 1 & $"$ \\
\hline & S. isangi & 6,$7 ; \mathrm{d} ; 1,5$ & 1 & $"$ \\
\hline C3 & S. comalitis & 8,$20 ; z 4, z 23 ;(z 6)$ & 1 & $"$ \\
\hline \multirow[t]{2}{*}{ D1 } & S. durban & 9,$12 ; a ; e, n, z 15$ & 1 & $"$ \\
\hline & s. saarbmoken & $\underline{1}, 9,12 ; a ; I, 7$ & 1 & $"$ \\
\hline \multirow[t]{3}{*}{ EI } & S. vejie & 3,$10 ; e, h ; I, 2$ & 1 & $"$ \\
\hline & S. воuza & 3,$10 ; d ; e, n, x$ & 1 & 11 \\
\hline & S. muenster & 3,$10 ; e, h ; 1,5$ & 1 & $"$ \\
\hline E2 & S. new-bmanswick & 3,$15 ; 1, v ; I, 7$ & 2 & $"$ \\
\hline E4 & S. ngor & $1,3,19 ; 1, v ; 1,5$ & 2 & $"$ \\
\hline G1 & S. poona & $1,13,22 ; z ; I, 6(z 59)$ & 1 & $"$ \\
\hline G2 & s. havana & $\underline{1}, 13,23 ; f, g,(s) ;-$ & 2 & $"$ \\
\hline \multirow[t]{3}{*}{ I } & S. nottingriam & $16 ; d ; e, n, 215$ & 2 & \\
\hline & S. welikade & $16 ; 1, v ; I, 7$ & 2 & $"$ \\
\hline & S. gaminara & $16 ; d ; 1,7$ & 2 & $"$ \\
\hline \multirow[t]{2}{*}{$\mathrm{J}$} & S. $20 d e$ & $17 ; r ; 1,2$ & 1 & \\
\hline & $S$. & $17 ; b ; e, n, z 15$ & 1 & \\
\hline 0 & S. adelatde & $35 ; f, g ;-$ & 1 & $"$ \\
\hline $\mathbf{R}$ & S. johannesburg & $\underline{I}, 40 ; b ; e, n, x$ & 2 & $"$ \\
\hline $\mathrm{v}$ & S. 2 arwra & $44 ; k ; e, n, z 15$ & 1 & \\
\hline W & S. tomow & $45 ; g, m,(s) ;-$ & 1 & \\
\hline
\end{tabular}

Ajouter dans la colonne « Sérotype n, après $S$. lodé $: S$. bignona. 
entérobactéries (Institut Pasteur de Dakar) sont alors toutes dirigées sur le Centre international des Salmonelles (Institut Pasteur de Paris, Professeur LE MINOR) pour confirmation et étude complète.

\section{RẾSULTATS}

Les résultats détaillés figurent dans les tableaux I et II.

Chez le mouton, à partir de 1108 prélèvements, 53 souches de Salmonella sont isolées, appartenant à 37 sérotypes. Le taux d'infection s'élève à $4,7 \mathrm{p}$. 100. Un sérotype nouveau est découvert (groupe 52), il répond à la formule antigénique suivante $52 ; \mathrm{e}, \mathrm{h} ; \mathrm{I}, 7$ et a reçu l'appellation de Salmonella derklé (nom d'un des faubourgs de Dakar).

Chez la chèvre, à partir de 1018 prélèvements, 37 souches sont isolées appartenant à 27 sérotypes. Le taux d'infection s'élève à 3,6 p. 100 . 2 sérotypes nouveaux sont découverts (groupe J), de formule antigénique $17 ; \mathrm{r} ; \mathrm{I}, 2$ et $17 ; \mathrm{b}$; e, n, z 15. Ils ont reçu le nom de Salmonella lodé (lieu-dit du Ferlo, proche du forage de Tatki, situé à environ $60 \mathrm{~km}$ au sud de Richard-Toll) et de $S$. bignona (ville de Casamance).

43 sérotypes ont déjà fait l'objet d'au moins une observation chez l'homme au Sénégal.

$S$. lawra, $S$. ruiru et $S$. tornow sont isolées pour la première fois. $S$. denver, $S$. redba et $S$. nottingham, jamais encore signalées chez l'homme au Sénégal, le sont pour la seconde fois chez l'animal (agame et chauve-souris lors du premier isolement).

\section{DISCUSSION}

Les taux d'infection obtenus à Dakar sont assez voisins de ceux observés au Zaire et au Soudan. Les porcs, abattus et étudiés dans les mêmes conditions, présentent une contamination bien supérieure ; d'après CHAMBRON et collab. (2), 18,9 p. 100 se révèlent être des infectés latents. En ce qui concerne les différents sérotypes mis en évidence, les points suivants doivent être soulignés :

1) Salmonella abortus ovis, responsable dans d'autres parties du monde d'enzooties d'avortements, en Iran par exemple (18), n'a pas été observée, chez les porteurs sains, mais $S$. typhimurium ( 2 moutons), $S$. enteritidis (1 mouton), $S$. virchow (3 moutons, 1 chèvre) et $S$. w'aycross
(4 moutons) sont isolées. Ces quatre derniers sérotypes présentent une fréquence non négligeable chez l'homme;

2) Aucun autre sérotype responsable d'affections humaines graves n'est trouvé (*). On peut donc logiquement penser avec VAN PEE et MAZIBO (19) que les viandes de mouton et de chèvre ne constituent pas une source d'in-' fection importante pour les salmonelloses humaines majeures au Sénégal ;

3) Toutefois, 43 sérotypes, isolés des petits ruminants, ont déjà été rencontrés chez l'homme à l'occasion de coprocultures effectuées dans les centres hospitaliers dakarois. La contamination des ganglions mésentériques par des sérotypes " rares » signe la contamination du contenu intestinal, donc celle des toisons (6), des peaux, des tables d'abattage, des instruments de boucherie et de triperie, etc... et en définitive de la viande. Le rôle de ces sérotypes « rares» de Salmonella a déjà fait l'objet de discussions dans des publications précédentes $(1,2,4)$, aussi apparaît-il inutile de revenir sur cette question. Qu'il nous suffise de répéter qu'il est bien imprudent d'affirmer «qu'un pouvoir pathogène faible le restera à l'occasion des multiples passages que les souches subissent chez des espèces animales variées en cours d'infections latentes $»(*)$.

Ainsi, les petits ruminants apparaissent comme des « réservoirs » de Salmonella non négligeables et seul le respect des règles élémentaires d'hygiène peut éviter la contamination des carcasses au moment de l'abattage.

\section{REMERCIEMENTS}

Il nous est particulièrement agréable de remercier ici, le Professeur LE MINOR, Directeur du Centre international des Entérobactéries à l'Institut Pasteur de Paris, qui a bien voulu se charger, comme à l'habitude, du contrôle et de l'étude complète des souches qui lui ont été adressées.

(*) A titre indicatif, le Service de Bactériologie de l'Institut Pasteur de Dakar a isolé chez l'homme en 1974, 168 souches de $S$. typhi (dont 136 par hémoculture), 158 souches de $S$. ordoñez (dont 133 par coprocultures), 30 souches de $S$. typhimurium (dont 21 par hémoculture, 7 par coproculture et 2 du liquide céphalo-rachidien), 7 souches de $S$. virchow (3 par hémoculture, 4 par coproculture), 10 souches de $S$, waycross (coproculture). S. havana, isolée une fois en 1974, arrivait au second rang en 1971 avec 85 isolements. Ce dernier fait illustre le changement de fréquence dans le temps des sérotypes observés chez l'homme. 


\title{
SUMMARY
}

Salmonella serotypes isolated in sheep and goats slaughtered in Dakar

A survey on "reservoirs» of Salmonella in small ruminants slaughtered in Dakar abattoir is carried out.

Mesenteric lymph nodes of 1108 sheep and 1018 goats are collected. After enrichment in selenite broth, 90 strains are isolated belonging to 52 serotypes. 2 new serotypes are discovered : $S$. derklé, $S$. lodé and $S$. bignona, and 3 serotypes reported for the first tıme in Senegal : $S$. lawra, $S$. ruiru and $S$. tornow. The infection rate raises to 4,7 p. 100 in sheep and 3,6 p. 100 in goats. Those results are closely related to those obtained in other african countries.

The incidence of healthy carriers of Salmonella, among small ruminants, on public hygiene is discussed.

\section{RESUMEN}

\begin{abstract}
Suerotipos de Salmonella aislados en los pequeños rumiantes matados en Dakar
Se efectua una encuesta sobre la presencia crónica de Salmonella en los pequeños rumiantes matados en el matadero de Dakar. Se recogen los ganglios mesentéricos de 1108 ovinos y de 1018 cabrunos. Después de enriquecimiento en medio selenita, se aislan 90 cepas perteneciendo a 52 suerotipos. Se descubre tres suerotipos nuevos : $S$. derkle, $S$. lodé y $S$. brgnona y 3 suerotipos encontrados por primera vez en Senegal : $S$. lawra, $S$. ruiru y $S$, tornow. La tasa de infección es de 4,7 p. 100 en la oveja y de 3,6 p. 100 en la cabra. Los dichos resultados son cercanos de los obtenidos en otros paises africanos.

Se discute la incidencia de la presencia crónica de Salmonella en los pequeños rumiantes sobre higiene pública.
\end{abstract}

\section{BIBLIOGRAPHIE}

1. CHAMBRON (J.), DOUTRE (M. P.), SARRAT (H.) et MARTEL (J.). Les salmonelloses au Sénégal. Importance des rapaces anthropophiles de la région du Cap-Vert en tant que réservoir de Salmonelles. Rev. Elev. Méd. vét. Pays trop., 1971, 24 (1) : 9-18.

2. CHAMBRON (J.), MARTEL (J. L.), SARRAT (H.) et DOUTRE (M. P.). Isolement de 28 souches de Salmonella à partir de ganglions mésentériques de porcs sains abattus à Dakar. Rev. Elev. Méd. rét. Pays trop., 1971, 24 (4) : 497-504.

3. CORRADINI (L.) et TOMASINI (A.). Ricerca di Salmonelle in agnelli macellati regolarmente. Vet. ital., 1969, 20 (5-6) : 351-353.

4. DOUTRE (M. P.) et SARRAT (H.). Sérotypes de salmonelles 1solées chez les chiroptères frugivores et insectıvores du Sénégal. Importance épıdémiologıque. Rev. Elev. Méd. vét. Pays trop., 1973, 26 (3) : 279-287.

5. GOTZE (U.). Latente Salmonella-Infektionen bet Schlachttieren (Untersuchungen an Schafen. Pferden und Kälbern). Zbl. Veter.-Med. B. 1968, 15 (8) : 878-894.

6. GRAU (F. H.) et SMITH (M. G.). Salmonella contamination of sheep and mutton carcasses related to pre-slaughter holding conditions. J. appl Bact., 1974,37 (1): 111-116.

7. GUPTA (P. D.). Incidence of Salmonella in beef and goat meat in West Bengal and its public health importance. Indian $J$. anim. Hith, 1974, 13 (2) : 161163.

8. JANAKIRAMAN (D.) et RAJENDRA (M. P.) The significance of isolation of Salmonella from goats used for the production of freeze-dried rinderpest goat tissue vaccine. Indian J. anim. Sci., 1973, 43 (3) : 220-223.

9. JOHNSON (R. H.). Notes on some west african salmonelloses. Bull. epiz. Dis. Afr., 1958, 6 (3) : 249-253.

10. KHAN (A. Q.). Salmonella infections in healthy sheep and goats in the Sudan. Bull. epiz. Dis. Afr., 1970,18 (2) : 117-122.

11. KUMAR (S.), SAXENA (S. P.) et GUPTA (B. K.). Carrier state of Salmonella in sheep and goats and its public health significance. $J . H y g ., 1973,71$ (1): 43-47.

12. LE MINOR (L.), VIGIER (M.), THOME (M.), CHARIE-MARSAINES (C.) et PERREAU (P.). Six nouveaux sérotypes de Salmonella isolés à FortLamy (Tchad). Ann. Inst. Pasteur, 1963, 104 (6) : 830-833.

13. MANICKAM (R.) et VICOTR (D. A.). A study on the occurence of Salmonella in abattoir carcasses. Indion vet. J., 1975, 52 (1) : 44-47.

14. NAMIN (P.). Contribution à l'étude épidémiologique des salmonelloses. Incidence des porteurs sains de Salmonella chez les chèvres en lran. Thèse. Doct. vét. Lyon, 1975, n" 67.

15. OLA-OJO (M.). Enquête sur la présence des Salmonellae caprines et canines au Nigeria Bull. epiz. Dis. Afr., 1974, 22 (1): 33-35.

16. POPOVA (P. P.). Réservoirs of Salmonella in central Kazakhstan (sheep, swine, rodents, birds). Zhurn. Mikrobiol. Epidem. Immunobiol., 1974 (3) : 137-138.

17. TADJEBAKHCHE (H.) et NAMIN (P.). Incidence des porteurs sains de Salmonella chez les chèvres en Iran. Rev. Méd. vét., 1974, 125 (12) : 1469-1474.

18. TADJEBAKHCHE (H.), DESLIENS (M.) et HEDJAZI (M.). Etude bactériologique d'enzooties d'avortements causés par Salmonella abortus ovis en Iran. Rev. Méd vét., 1971, 122 (6) : 621-628.

19. VAN PEE (W.) et MAZIBO (F.). Le rôle des viandes de boucherie dans l'épıdémiologie des salmonelloses humaines à Kinshasa Ann. Soc. Belg. Méd. trop., 1974, 54 (6) : 451-455.

20. VARELA (G.), VELASCO (R.) et ROMAN (A.). Salmonellas aisladas en intestinos de cabras del rastro de la ciudad de Mexico. Revta Invest. Salud publ., Mexico, 1970, 30 (3) : 237-241.

21. VIKTOR (T.) et VAN OYE (E.). Importance des animaux de boucherie comme propagateurs de salmonelloses humaines à Stanleyville. Ann. Soc. belge Méd. trop., 1955, 35 (6) : 825-832.

22. ZWART (D.). Notes on Salmonella infections in animals in Ghana. Res. vet. Sci., 1962, 3 (1) : 460-469. 